\title{
miR-10a-5p Inhibits the Differentiation of Goat Intramuscular Preadipocytes by Targeting KLF8 in Goats
}

\author{
Qing $X u^{1,2,3}$, Yong Wang ${ }^{1,2,3}$, Xin $L i^{1,2,3}, Y u{ }^{1,2}$, Yanyan $L i^{1,3 *}$, Jiangjiang $Z h u^{1,2}$ and \\ Yaqiu Lin ${ }^{1,2,3 *}$ \\ ${ }^{1}$ Key Laboratory of Qinghai-Tibetan Plateau Animal Genetic Resource Reservation and Utilization of Education Ministry, \\ Southwest Minzu University, Chengdu, China, ${ }^{2}$ Key Laboratory of Qinghai-Tibetan Plateau Animal Genetic Resource Reservation \\ and Exploitation of Sichuan Province, Southwest Minzu University, Chengdu, China, ${ }^{3}$ College of Animal Science and Veterinary, \\ Southwest Minzu University, Chengdu, China
}

OPEN ACCESS

Edited by:

Xiao $\mathrm{Li}$,

Northwest $A$ and F University, China

Reviewed by:

Zhuanjian Li,

Henan Agricultural University, China

Carmela De Marco,

Magna Grãcia University of Catanzaro,

Italy

*Correspondence:

Yaqiu Lin

linyq1999@163.com

Yanyan Li

liyanyan@swun.edu.cn

Specialty section:

This article was submitted to

Cellular Biochemistry,

a section of the journal

Frontiers in Molecular Biosciences

Received: 25 April 2021

Accepted: 13 July 2021

Published: 13 August 2021

Citation:

Xu Q, Wang Y, Li X, Du Y, Li Y, Zhu J and Lin $Y$ (2021) miR-10a-5p Inhibits

the Differentiation of Goat Intramuscular Preadipocytes by

Targeting KLF8 in Goats.

Front. Mol. Biosci. 8:700078.

doi: $10.3389 /$ fmolb.2021.700078
Intramuscular fat contributes to the improvement of meat quality of goats. MicroRNAs (miRNAs) have been reported to regulate adipocyte differentiation and maturation. The aim of our study was to clarify whether miR-10a-5p regulates goat intramuscular preadipocyte (GIPC) differentiation and its direct downstream signaling pathway. GIPCs were isolated from longissimus dorsi, whose miR-10a-5p level was measured at different time point of differentiation induction. Adipogenic differentiation of the GIPCs was evaluated by Oil Red $O$ and BODIPY staining, and the expression changes of adipogenic genes like ACC, ATGL, CEBP $\beta$, PPAR $\gamma$, etc. Related mechanisms were verified by qPCR, a bioinformatic analysis, a dual-luciferase reporter assay, overexpression, and siRNA transfection. Oil Red $O$ and BODIPY staining both with adipogenic gene detection showed that miR-10a-5p suppressed the accumulation of lipid droplets in GIPCs and inhibited its differentiation. The dual-luciferase reporter assay experiment revealed that miR-10a-5p regulates GIPC differentiation by directly binding to KLF8 3'UTR to regulate its expression. Thus, the results indicated that miR-10a-5p inhibits GIPC differentiation by targeting KLF8 and supply a new target for fat deposition and meat quality improvement.

Keywords: MiR-10a-5p, KLF8, goat, intramuscular preadipocytes, differentiation

\section{INTRODUCTION}

Intramuscular fat (IMF) is deposited in skeletal muscle fibers (Hocquette et al., 2010). IMF can be used to store energy and functions during exercise, but an excessive accumulation of IMF in the muscle is related to many diseases in people, such as diabetes, insulin resistance, lipodystrophy, etc. (Goodpaster et al., 2000; Shoelson et al., 2007; Tada et al., 2020; Yamada et al., 2020). The IMF content in animals is not only an important index of high quality of meat but also an important character of good germplasm. IMF is an important factor for meat quality which is connected with meat tenderness, meat flavor, and color (Wood et al., 2004; Chen and Sui, 2018; Liu et al., 2019). The number and size of intramuscular adipocytes mainly determine the IMF contents, and intramuscular adipocytes are very important because they provide sites for later marbling fat deposition. However, the mechanism of preadipocyte differentiation still needs to be further investigated.

MicroRNAs (miRNAs or miRs) are small noncoding RNAs; several studies suggested that they are the negative regulator over the process of target gene expression through degrading mRNAs or inhibiting the translation of mRNAs (Mourelatos, 2008; Li et al., 2014; Schreck et al., 2017; Lin et al., 
2019; van der Kwast et al., 2020). Recently, more and more studies have proved the significance of miRNAs in regulating adipogenic differentiation. However, roles of miRNAs in GIPC differentiation shift fate are still unclear.

In the current study, we explored the action of miR-10a-5p in GIPC differentiation as well as mechanisms during the process. In general, we demonstrated that miR-10a-5p expression was significantly changed during preadipocyte differentiation in goats. MiR-10a-5p inhibits GIPC differentiation by targeting KLF8. Therefore, our conclusion supplied a new idea and theoretical basis for the basic research of improving the quality of meat.

\section{MATERIALS AND METHODS \\ Isolation and Cell Culture of Goat Intramuscular Preadipocytes}

The 7-day-old Jianzhou Daer male goats $(n=3)$ were used as an experimental model. The GIPCs were isolated and cultured, as we previously described (Xu et al., 2018; Ma et al., 2021). Briefly, the isolated longissimus dorsi muscles of goats were washed with PBS for three times and minced and then were digested with an equal volume of collagenase type II at $37^{\circ} \mathrm{C}$ for $2 \mathrm{~h}$ in a shaking water bath every $5 \mathrm{~min}$. Steel mesh filters of 200 and $400 \mu \mathrm{m}$ were utilized to isolate digested cells. The rinsed filtrated cells by $\mathrm{DMEM} / \mathrm{F} 12$ medium were centrifuged twice at $2000 \mathrm{r} / \mathrm{min}$ for 5 min to collect sediment clumps and then the supernatant was discarded. The viable cells were resuspended in DMEM/F12, including $10 \%$ fetal bovine serum and plated in $25-\mathrm{cm}^{2}$ flask in a $5 \% \mathrm{CO}_{2}$ atmosphere at $37^{\circ} \mathrm{C}$ for subsequent culture. Experimentation on goats performed in the present study had been given prior approval by the Ethics Committee of Southwest Minzu University under permit no. SMU20160108, and all of the methods were performed according to the guidelines and regulations.

\section{Preadipocyte Differentiation Induction}

We induced GIPCs adipogenic differentiation in vitro as before (Xu et al., 2018). Briefly, adipogenesis induction medium [MEM/ F12 containing $10 \% \mathrm{FBS}$ and $50 \mu \mathrm{mol} \bullet \mathrm{L}^{-1}$ oleic acid (Sigma)] was used to culture GIPCs in 12-well plates with a density of $1 \times 10^{6}$ cells per well for the required time point. We changed the culture medium every other day. After induction, Oil Red $\mathrm{O}$ staining and BODIPY staining were used to distinguish mature adipocytes from preadipocytes during the process of culture.

\section{Oil Red $O$ and BODIPY Staining}

Adipogenic differentiation of the GIPCs was assessed by Oil Red O or BODIPY staining as previously described (Xu et al., 2018). The GIPCs were washed with PBS and fixed in $10 \%$ formaldehyde for $10 \mathrm{~min}$, then washed with PBS, and stained using the Oil Red $\mathrm{O}$ or BODIPY working solutions for $20 \mathrm{~min}$. The cells were then observed and photographed after washing. After photographing,

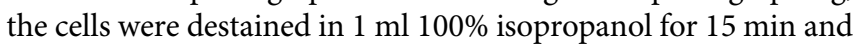
the Oil Red signal was quantified by measuring the absorbance at $490 \mathrm{~nm}(\mathrm{OD} 490)$ as a semi-quantitative assessment method to determine the extent of differentiation. The stained area of Oil Red O or BODIPY staining was measured using Image $(\mathrm{NIH}$, Bethesda, MD, United States).

\section{qRT-PCR}

Total RNA from cells was extracted using the TRIzol reagent (TaKaRa) according to the manufacturer's protocol. The mRNAs were reverse transcribed using the RevertAid First Strand cDNA Synthesis Kit (Thermo) according to the protocol. Then, amplification reactions were performed using amplification primers with the SYBR Green PCR Master Mix (TaKaRa); the reaction volumes were $20 \mu \mathrm{l}$. Then $1 \mu \mathrm{l}$ of cDNA was applied in every set of experiment. The mRNA expression levels were standardized to UXT or U6. Information on primers for qPCR is listed in Table $\mathbf{1}$.

\section{Transfection}

Small interfering RNA (siRNA) against KLF8, 5'CAGACUCUUGUAGUGUCCACUUCAAdTdT-3' was synthesized by Invitrogen. KLF8 expression plasmid was constructed by inserting expanded KLF8 cDNA (KX247671) fragments into pcDNA3.1 vector (sense primer sequence: 5'CGGGGTACCATGGATGAACTCATAAACAACT-3', antisense primer sequence: 5'-ATAAGAATGCGGCCGCTTACA CGGTGTCATGGCGC-3'). The KLF8 interference (designated as siKLF8) or negative control (siNC) GIPCs were constructed using siRNA. The KLF8 overexpression (designated as KLF8) GIPCs were constructed using an expression plasmid, and the control cells for the KLF8 overexpression group were designated as a vector. Cells had been pre-cultured for $2 \mathrm{~h}$ in a serum-free medium for transfection. Then plasmid or siRNA was introduced into the cells using a Lipofectamine 3000 transfection reagent, in accordance with the manufacturer's instruction (Invitrogen, Carlsbad, United States).

The miR-10a-5p mimics (designated as mimics: UACCCU GUAGAUCCGAAUUUGU), an inhibitor (designated as inhibitor: ACAAAUUCGGAUCUACAGGGUA), and a respective negative control (designated as mock: UUGUAC UACACAAAAGUACUG, NC: CAGUACUUUUGUGUAGUA CAA) (Genepharma, Shanghai, China) as needed were transfected into the GIPCs by Lipofectamine 3000 (Invitrogen, Carlsbad, United States) and opti-MEM (Gibco BRL Co., LTD) culture medium according to the manufacturer's instruction.

After 12-h transfection, the original medium was replaced by a fresh differentiation medium to induce GIPC differentiation. After 48-h induction, the cells were used for Oil red $\mathrm{O}$ or BODIPY staining or collected to extract RNA for qPCR detection.

\section{Luciferase Reporter Assay}

For the luciferase reporter assay, the 3'UTR of KLF8 containing the wild or mutant miR-10a-5p target sites was cloned using primers with NotI and XhoI (Thermo, MA, United States) cleavage sites. The wild or mutant type 3'UTR fragment was inserted into the corresponding site of the psiCHECK vector and then co-transfected into $293 \mathrm{~T}$ cells with miR-10a-5p mimics/ mock. After $48 \mathrm{~h}$ transfection, the cells were harvested and the Dual-Luciferase Reporter Assay System Kit (Promega, Madison, 
TABLE 1 | The sequences information of specificity primers.
Gene/miRNA (Accession number in GenBank)

ACC (XM_018064169.1)

ATGL (NM_001285739.1)

AP2 (NM_001285623.1)

CEBPa (XM_018062278)

CEBPß (XM_018058020.1)

DGAT2 (NM_001314305.1)

GLUT4 (NM_001314227.1)

FASN (NM_001285629.1)

HSL (XM_018062484.1)

LPL (NM_001285607.1)

PPARy (NM_001285658)

Pref1 (KP686197.1)

SREBP1 (NM_001285755)

KLF8 (KX247671)

UXT (XM_005700842.2)

U6 (NR_138,085.1)

miR-10a-5p
Sequence

GGAGACAAACAGGGACCATT ATCAGGGACTGCCGAAAC GGTGCCAATATCATCGAGGT CACACCCGTGGCAGTCAG TGAAGTCACTCCAGATGACAGG TGACACATTCCAGCACCAGC CCGTGGACAAGAACAGCAAC AGGCGGTCATTGTCACTGGT CAAGAAGACGGTGGACAAGC AACAAGTTCCGCAGGGTG CAATAGGTCCAAGGTAGA GAAGC

ACCAGCCAGGTGAAGTAGAGC TGCTCATTCTTGGACGGTTCT CATGGATTCCAAGCCTAGCAC TGTGCAACTGTGCCCTAG GTCCTCTGAGCAGCGTGT AGGGTCATTGCCGACTTCC GTCTCGTTGCGTITGTAGTGC TCCTGGAGTGACGGAATCTGT GACAGCCAGTCCACCACGAT AAGCGTCAGGGTTCCACTATG GAACCTGATGGCGTTATGAGAC CCGGCTTCATGGATAAGACCT GCCTCGCACTTGTTGAGGAA AAGTGGTGGGCCTCTCTGA GCAGGGGTTTCTCGGACT GACTACAGCAAGAACCAG

CAGC

CTCCTGTATGGATTCTGCGGT GCAAGTGGATITGGGCTGTAAC ATGGAGTCCTTGGTGAGGTTGT TGGAACGCTTCACGAATITGCG GGAACGATACAGAGAAGA TTAGC

CAGCTGTACCCTGTAGATCCGA GTGCAGGGTCCGAGGT
WI, United States) was used for detecting dual-luciferase activity, according to the manufacturer's instructions.

\section{Statistical Analysis}

All data were presented as "mean \pm SD." The variance of data was analyzed by SPSS 17.0, followed by Duncan's multiple comparisons test. ${ }^{*}$ indicates the $p$ values were $<0.05, \geq 0.01$, whereas ${ }^{* *}$ indicates $p$ values $<0.01$. All experiments in our study were carried out for three times at least.

\section{RESULTS}

\section{miR-10a-5p Expression Changed During GIPCs Differentiation}

MicroRNAs have been reported to regulate adipogenic differentiation (Hamam et al., 2015; Tang et al., 2017; Ai et al., 2019; Li et al., 2019); however, the role of miR-10a-5p in goat intramuscular adipogenesis has not been reported. The differential expression of miR-10a-5p after GIPC differentiation has been observed by miRNA sequencing technology in our previous study (data not shown). In order to clarify the role of miR-10a-5p in the differentiation of GIPCs, we first isolated intramuscular preadipocytes from goat longissimus dorsi and induced them to adipogenic differentiation. Oil red $\mathrm{O}$ staining was used to ascertain the extent of differentiation; our obtained results showed that the lipid droplets accumulation increased with the extension of induction time, and the GIPCs were differentiated completely after 60-h induction (Figure 1A). Then qRT-PCR was implemented to research the role of miR$10 a-5 p$ in adipogenic differentiation of GIPCs. The test result showed an obvious alteration in the expression of miR-10a-5p during differentiation when compared to $0 \mathrm{~h}$ (Figure 1B). All above indicate that $\mathrm{miR}-10 \mathrm{a}-5 \mathrm{p}$ may regulate the adipogenic differentiation of GIPCs.

\section{miR-10a-5p Inhibits the Adipogenic Differentiation of GIPCs}

To clarify the effect of miR-10a-5p on GIPC adipogenic differentiation, miR-10a-5p mimics (named as miR-10a-5p) or its control (named as mock) was transfected to GIPCs to overexpress miR-10a-5p. The expression of miR-10a-5p in GIPCs increased $\sim 10,000$ times caused by mimic transfection than the same amount of control vector transfected cells (Figure 2A). Preadipocyte differentiation is associated with lipid droplets accumulation in the cells. Oil red $\mathrm{O}$ and BODIPY stainings were utilized as lipid droplet detection methods. In our study, after miR-10a-5p overexpression, the lipid droplet accumulation was significantly decreased in the group of mimic-transfected cells than the control group (Figures 2B-E). Oil red $\mathrm{O}$ and BODIPY staining results showed about $20-30 \%$ reduction of lipid droplets with miR-10a-5p overexpression (Figures 2B-E). Additionally, there are representative of the genes upregulated during the differentiation process from preadipocytes to mature adipocytes. Indeed, the mRNA expression levels of representative genes AP2, DGAT2, FASN, HSL, LPL, and Prefl were obviously inhibited due to the overexpression of miR-10a-5p in GIPCs (Figure 2F). All these results implied that miR-10a-5p may weaken the adipogenic differentiation of GIPCs.

For further validating the suppression effect of miR-10a-5p on GIPC adipogenic differentiation, miR-10a-5p inhibitor (named as inhibitor) or its control (named as NC) was transfected to GIPCs to silence miR-10a-5p expression. About $80 \%$ of the interference efficiency was caused by inhibitor transfection compared to the NC group (Figure 3A). Oil red $\mathrm{O}$ and BODIPY staining results showed that compared with the control group, miR-10a-5p knockdown significantly promoted the about $10-20 \%$ of lipid droplet accumulation (Figures 3B-E). Coincidently, the mRNA levels of important markers of adipocyte differentiation like ACC, ATGL, CEBP $\beta$, GLUT4, HSL, PPAR $\gamma$, and Pref1 were upregulated due to the knockdown of miR-10a-5p in GIPCs (Figure 3F). Therefore, miR-10a-5p inhibits adipogenic differentiation of GIPCs. 
A

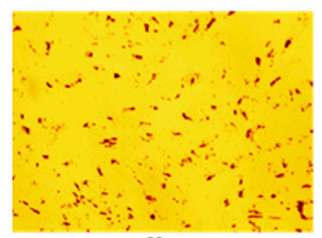

3h

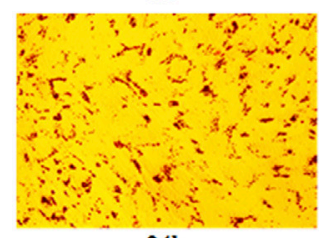

24h

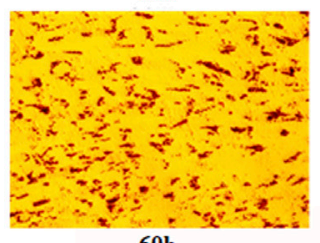

B

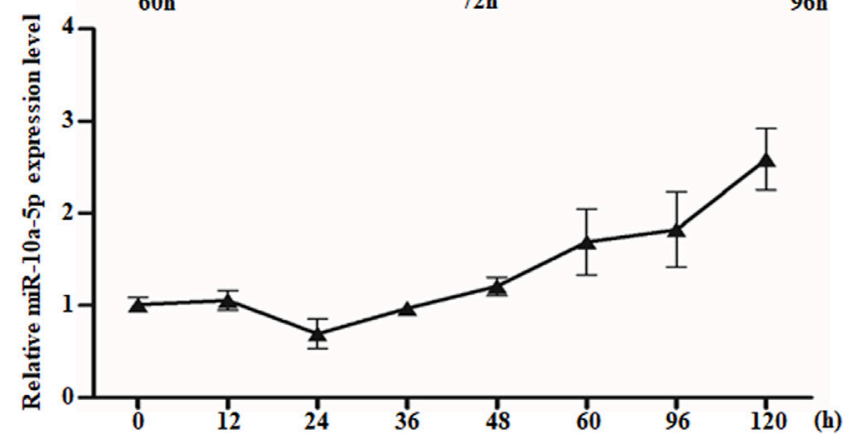

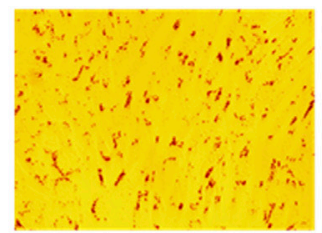

$6 \mathrm{~h}$

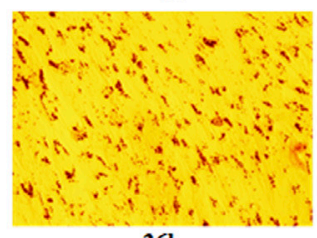

$36 \mathrm{~h}$
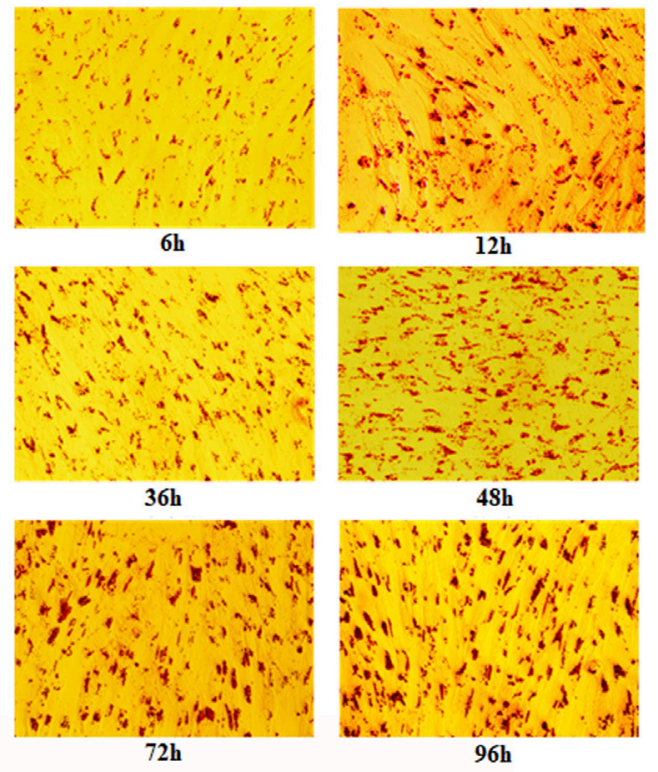

$12 \mathrm{~h}$

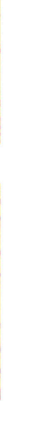

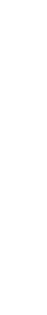

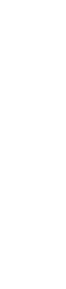


A

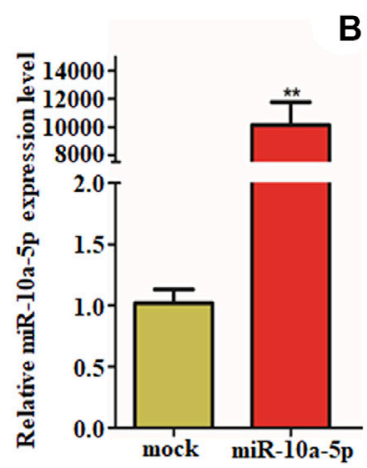

D
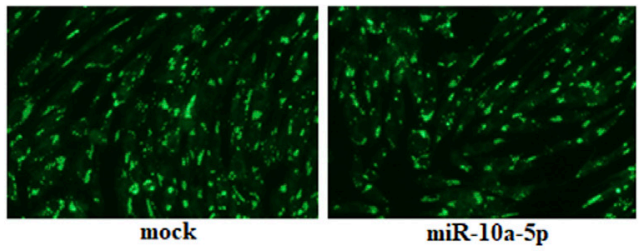

C
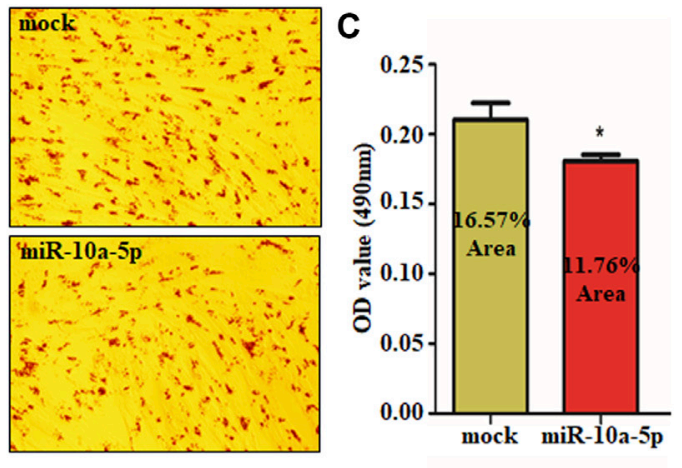

E

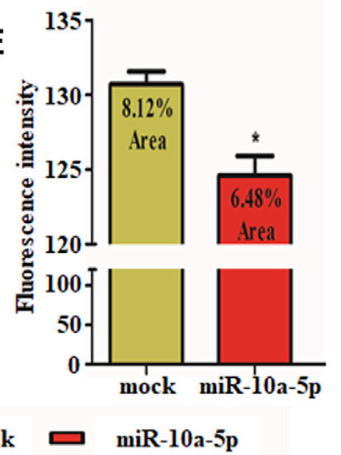

$\mathbf{F}$

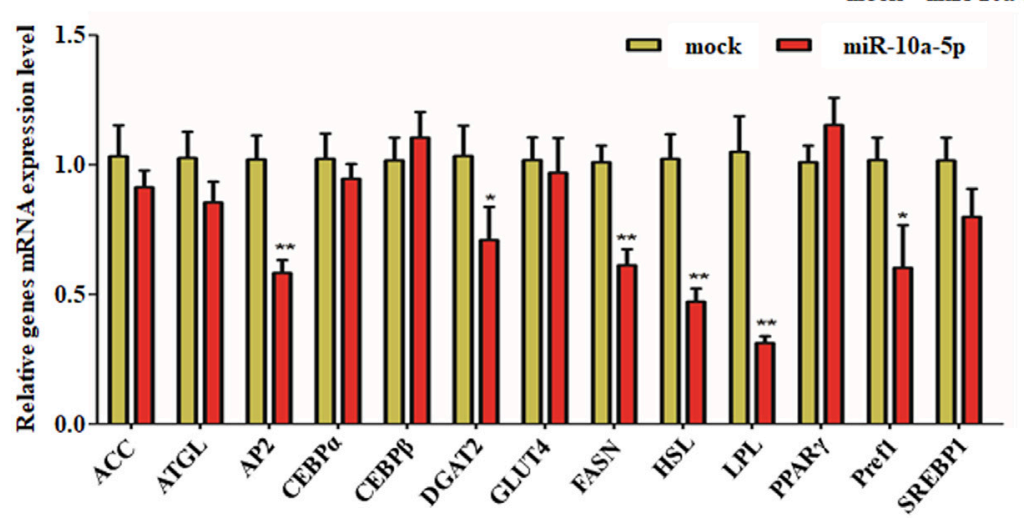

FIGURE 2 | Overexpression of miR-10a-5p inhibits GIPC differentiation. (A) qRT-PCR analysis of levels of miR-10a-5p expression in GIPCs with mimics or control transfected for 24 h. (B) Representative images of Oil Red O staining of GIPCs with miR-10a-5p mimics or control and (C) semi-quantitative assessment of Oil Red O content absorbance detection at $490 \mathrm{~nm}$. (D) Representative images of mature adipocytes stained with BODIPY and (E) stained area was measured using ImageJ. (F) qRT-PCR analysis of levels of genes expression in GIPCs with miR-10a-5p mimics or control. $\mathrm{N}>$ or $=3$, * indicates $p$ values $<0.05$ and $\geq 0.01$, ** indicates $p$ values $<0.01$.

adipogenic differentiation, we further explored the relationship between KLF8 expression and GIPCs differentiation. We first synthesized KLF8 siRNA (named as siKLF8 and its control named as siNC) and constructed its expression plasmid (named as KLF8 and its control named as vector). Both the expression plasmid and siRNA were effective that cells transfected with the KLF8 expression plasmid can upregulate its expression about 5,000 times, and KLF8 siRNA can downregulate its expression about 60 times (Figure 5A). Oil red $\mathrm{O}$ and BODIPY staining results showed that KLF8 overexpression could contribute to the lipid droplet accumulation; nevertheless, KLF8 knockdown can inhibit the lipid droplet accumulation when compared with each control group, respectively (Figures 5B-E). qPCR was used to detect relative adipogenic gene expression, and the crucial adipocyte differentiation markers DGAT2, ACC, and LPL were markedly promoted by KLF8 overexpression (Figure 5F). Also, LPL, PPAR $\gamma$, and C/EBP $\beta$ as adipocyte differentiation genes were obviously inhibited by KLF8 interference (Figure 5F). In summary, our results indicated that KLF8 confers GIPCs with more properties of adipogenic differentiation.

\section{DISCUSSION}

Preadipocytes differentiate into adipocytes, which improve the color and flavor of meat in animals. According to the results we obtained, we expounded that miR-10a-5p is involved in regulating GIPCs adipogenic differentiation. 


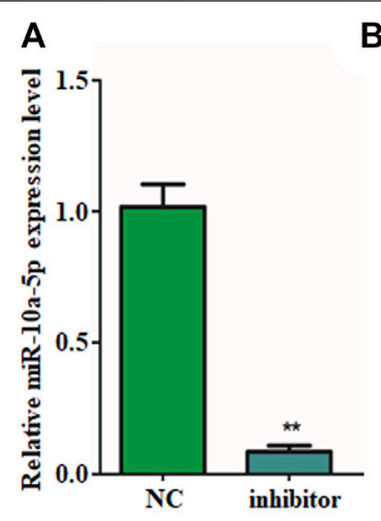

D
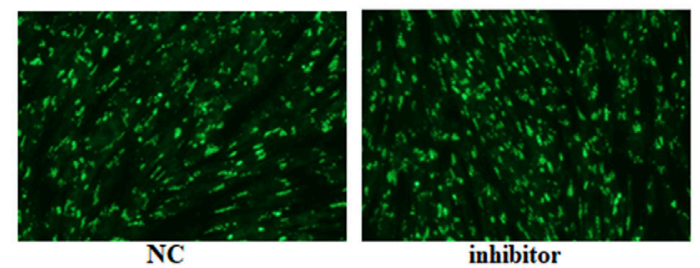

$\mathbf{F}$

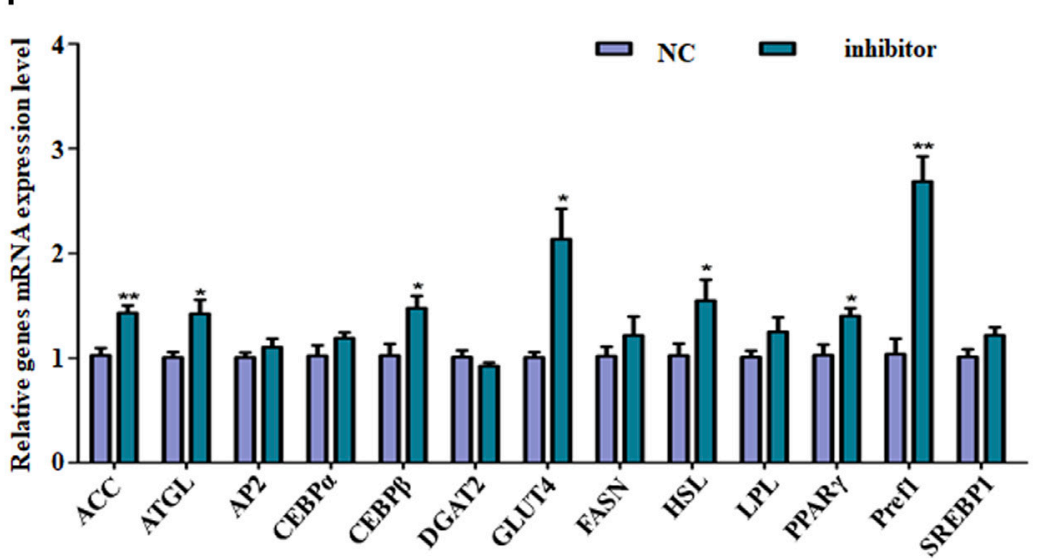

C
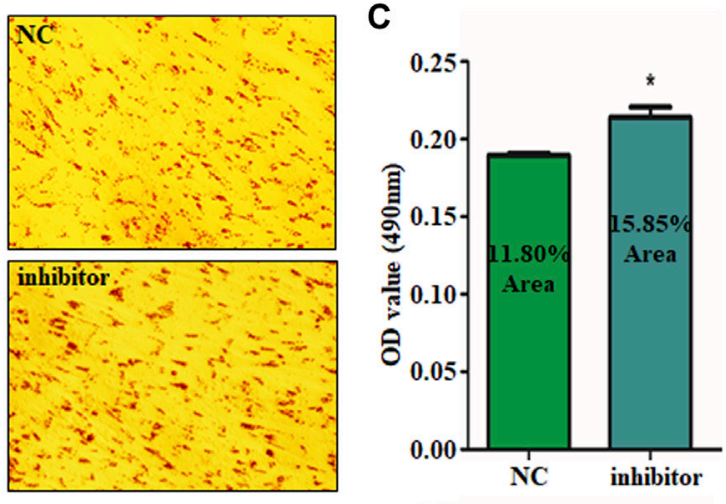

E

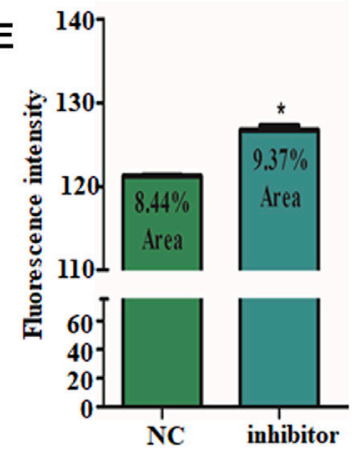

FIGURE 3 | Silence of miR-10a-5p promotes GIPC differentiation. (A) qRT-PCR analysis of levels of miR-10a-5p expression in GIPCs with inhibitor or control transfected for 24 h. (B) Representative images of Oil Red O staining of GIPCs with miR-10a-5p inhibitor or control and (C) semi-quantitative assessment of Oil Red O content absorbance detection at $490 \mathrm{~nm}$. (D) Representative images of mature adipocytes stained with BODIPY and (E) stained area was measured using ImageJ. (F) qRT-PCR analysis of levels of genes expression in GIPCs with miR-10a-5p inhibitor or control. $\mathrm{N}>$ or $=3$, * indicates $p$ values $<0.05$ and $\geq 0.01$, ** indicates $p$ values $<0.01$

miR-10a-5p directly targets the 3'UTR of KLF8 to inhibit its expression which promotes adipogenic differentiation. Furthermore, miR-10a-5p inhibits the accumulation of lipid droplets and the expression of relative adipogenic genes. Our results indicate that $\mathrm{miR}-10 \mathrm{a}-5 \mathrm{p}$ regulates alteration and lineage fate in GIPCs at the adipogenic differentiation process.

Accumulation of lipid droplets in the cells is associated with the differentiation of preadipocytes (Lee et al., 2017; Peng et al., 2018). This was verified by Oil red O and BODIPY staining in our study. Additionally, the differentiation of adipocytes is featured with genes expression alteration such as PPAR $\gamma, \mathrm{LPL}, \mathrm{HSL}$, and C/EBP, and so on (Rosen and MacDougald, 2006; Kim et al., 2015; Lee et al., 2017; Peng et al., 2018; Chen X. et al., 2020). Indeed, during the differentiation process from preadipocytes to mature adipocytes, ACC, ATGL, CEBP $\beta$, GLUT4, HSL, PPAR $\gamma$, and Pref1 are the representative upregulated genes. It is interesting that the adipogenic genes expression in $\mathrm{miR}$ 10a-5p overexpression GIPCs are not consistent with miR- 


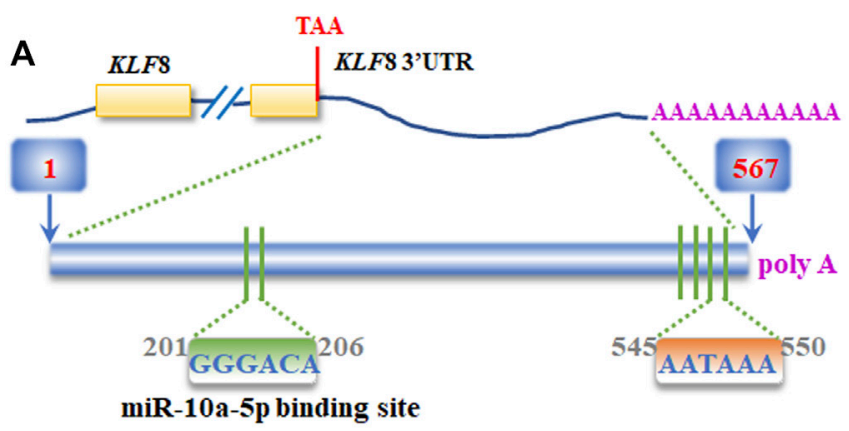

miR-10a-5p: --TACCCTGTAGATCCGAATTTGT--

IIIIII

KLFS 3'UTR WT: -AA GGGACAGAGTAGGAGTTAAA --

C

miR-10a-5p: --TACCCTGTAGATCCGAATTTGT--

III

KLF8 3'UTR MT: --AA GGGCTCGAGTAGGAGTTAAA --
B

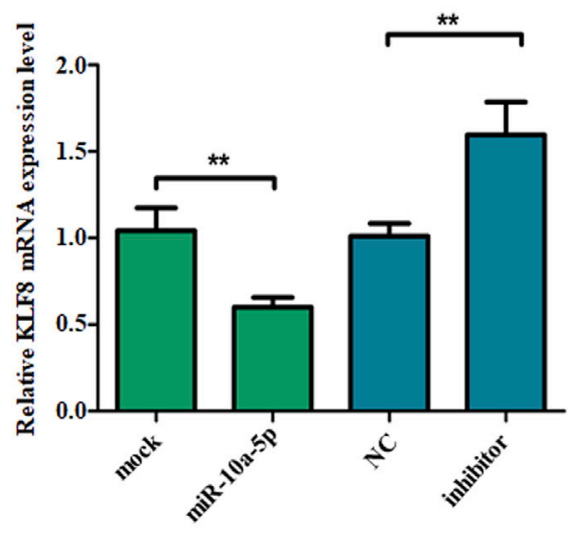

D

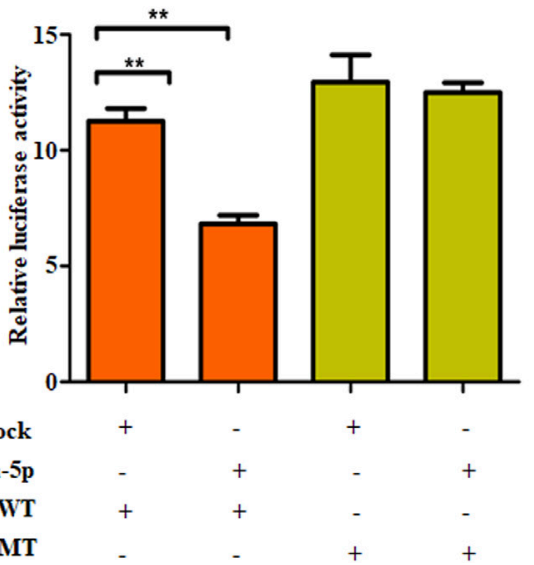

FIGURE 4 | miR-10a-5p directly bind to KLF8 3'UTR. (A) The predicted miR-10a-5p binding site in KLF8 3'UTR. (B) qRT-PCR analysis of levels of genes expression in GIPCs with miR-10a-5p mimics, inhibitor, or each control. (C) mutant miR-10a-5p binding site in KLF8 3'UTR. (D) Luciferase assay of transfected with wild-type or mutant KLF8 3'UTR plasmid in 293T cells. $\mathrm{N}>$ or $=3$ for B and $\mathrm{D}$, * indicates $p$ values $<0.05$ and $\geq 0.01,{ }^{* *}$ indicates $p$ values $<0.01$.

10a-5p knockdown cells (Figures 2F, 3F). KLF8 overexpression and knockdown cells showed the same phenomenon (Figure 5F). Here, we confirmed that KLF8 was the target of miR-10a-5p, but the knockdown of KLF8 causing the expression tendency of differentiation-related genes was not completely consistent with miR-10a-5p overexpression (Figures 2F, 5F). Also, the expression trend of differentiation-related genes did not present the same consistency in both KLF8 overexpression cells and miR10a-5p knockdown cells (Figures 3F, 5F). One possible explanation for this observed deviation is that the differentiation of GIPCs was regulated by more complex mechanisms, and this deviation might be referred to other regulation pathways for which further investigation would be need.

miRNAs are known as endogenous small noncoding RNAs that have been identified as gene expression posttranscriptional regulators, and miRNAs bind mainly to the target mRNA's 3' untranslated regions (UTRs), resulting in the blockade of mRNA translation or mRNA degradation
(Kerr et al., 2011; Callegari et al., 2013). Thus, miRNAs play vital roles in the differentiation and maturation of adipocytes, as shown in the findings of the present studies (Kato et al., 2012; Kobayashi et al., 2013; Miyoshi et al., 2014; Fujita et al., 2015a; Fujita et al., 2015b; Fujihara et al., 2015; Fujimori et al., 2015; Kato et al., 2016). Several miRNAs have been reported to contribute to lipid synthesis, metabolism, transportation, and storage. miR-10a-5p was reported to be relevant to proliferation, metastasis, invasive, drug resistance, inflammation, and other behaviors in cancer cells. In a previous investigation, miR-10a-5p was shown to restrain adipogenic differentiation in primary mouse preadipocytes. In the current study, up- or downregulation of $\mathrm{miR}-10 \mathrm{a}-5 \mathrm{p}$ was found to be involved in the differentiation of preadipocytes in goats. We also found that miR-10a-5p binds to the 3'UTR of KLF8 to perform its functions in an KLF8-dependent pathway. Taken together, our studies show that miR-10a-5p is critical for GIPC differentiation. 

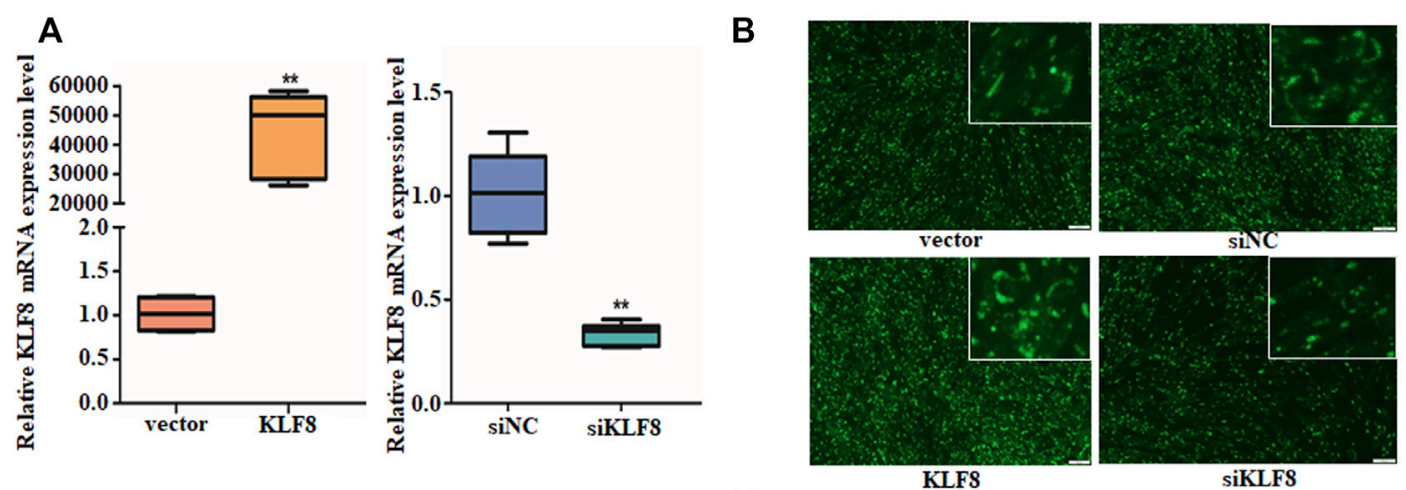

C
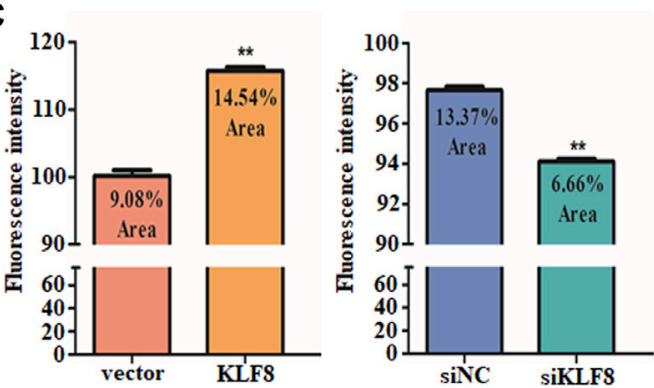

D
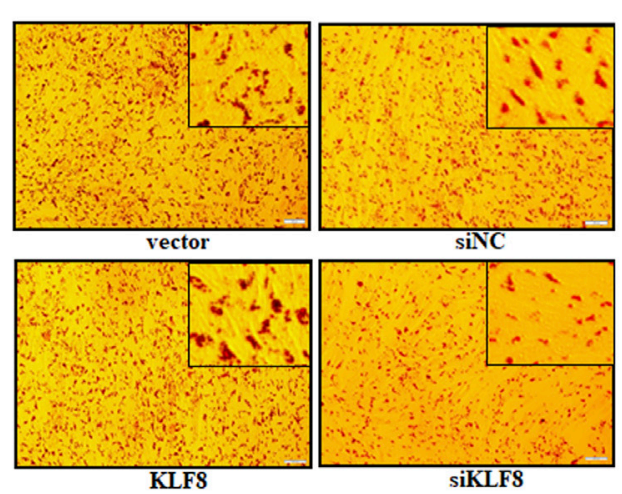

$\mathbf{F}$
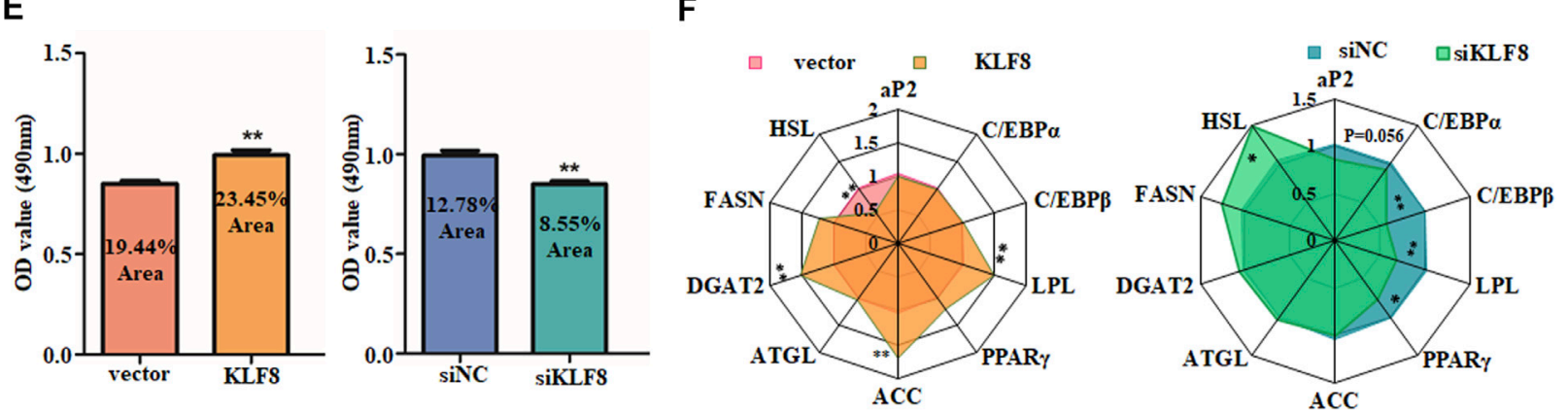

FIGURE 5|KLF8 promotes GIPC differentiation. (A) qRT-PCR analysis of levels of KLF8 with KLF8 siRNA, expression plasmid, or each control transfected for $24 \mathrm{~h}$. (B) Representative images of mature adipocytes stained with BODIPY and (C) stained area was measured using ImageJ. (D) Representative images of Oil Red O staining of GIPCs with miR-10a-5p inhibitor or control and (E) semi-quantitative assessment of Oil Red O content absorbance detection at $490 \mathrm{~nm}$. (F) qRT-PCR analysis of genes expression in GIPCs with KLF8 up- or downregulation, the result was showed by a network diagram. $\mathrm{N}>0 \mathrm{or}=3$ for $\mathrm{B}$ and $\mathrm{D},{ }^{*}$ indicates $p$ values $<0.05$ and $\geq 0.01,{ }^{* \star}$ indicates $p$ values $<0.01$.

\section{CONCLUSION}

Our results show that miR-10a-5p acts as an inhibitor of GIPC differentiation by targeting KLF8. This finding supplied a new target and possible mechanism for the basic research of meat quality improvement.

\section{DATA AVAILABILITY STATEMENT}

The raw data supporting the conclusions of this article will be made available by the authors, without undue reservation.

\section{ETHICS STATEMENT}

The animal study was reviewed and approved by the Ethics Committee of Southwest Minzu University.

\section{AUTHOR CONTRIBUTIONS}

Concept and design: QX, YW, XL, YD, YL, JZ, and YQL; development of methodology: QX, YL, YW, and YQL; 
acquisition of data: QX and YQL; analysis and interpretation of data: QX; writing, review, and/or revision of the manuscript: QX, YL, YW, and YQL; administrative, technical, or material support: YL, YW, and JZ; study supervision: YW and YQL.

\section{REFERENCES}

Ai, G., Meng, M., Wang, L., Shao, X., Li, Y., Cheng, J., et al. (2019). microRNA-196a Promotes Osteogenic Differentiation and Inhibit Adipogenic Differentiation of Adipose Stem Cells via Regulating $\beta$-catenin Pathway. Am. J. Transl Res. 11, 3081-3091.

Callegari, E., Elamin, B. K., Sabbioni, S., Gramantieri, L., and Negrini, M. (2013). Role of microRNAs in Hepatocellular Carcinoma: A Clinical Perspective. Onco Targets Ther. 6, 1167-1178. doi:10.2147/OTT.S36161

Chen, G., and Sui, Y. (2018). Production, Performance, slaughter Characteristics, and Meat Quality of Ziwuling Wild Crossbred Pigs. Trop. Anim. Health Prod. 50, 365-372. doi:10.1007/s11250-017-1441-2

Chen, J., Yang, Y., Li, S., Yang, Y., Dai, Z., Wang, F., et al. (2020). E2F1 Regulates Adipocyte Differentiation and Adipogenesis by Activating ICAT. Cells 9, 1024. doi:10.3390/cells9041024

Chen, X., Raza, S. H. A., Cheng, G., Ma, X., Wang, J., and Zan, L. (2020). Bta-miR376a Targeting KLF15 Interferes with Adipogenesis Signaling Pathway to Promote Differentiation of Qinchuan Beef Cattle PreadipocytesKLF15 Interferes With Adipogenesis Signaling Pathway to Promote Differentiation of Qinchuan Beef Cattle Preadipocytes. Animals 10, 2362. doi:10.3390/ ani10122362

Fujihara, S., Kato, K., Morishita, A., Iwama, H., Nishioka, T., Chiyo, T., et al. (2015). Antidiabetic Drug Metformin Inhibits Esophageal Adenocarcinoma Cell Proliferation In Vitro and In Vivo. Int. J. Oncol. 46, 2172-2180. doi:10.3892/ ijo. 2015.2903

Fujimori, T., Kato, K., Fujihara, S., Iwama, H., Yamashita, T., Kobayashi, K., et al. (2015). Antitumor Effect of Metformin on Cholangiocarcinoma: In Vitro and In Vivo Studies. Oncol. Rep. 34, 2987-2996. doi:10.3892/ or.2015.4284

Fujita, K., Iwama, H., Oto, T., Okura, R., Kobayashi, K., Takano, J., et al. (2015a). Galectin-9 Suppresses the Growth of Hepatocellular Carcinoma via Apoptosis In Vitro and In Vivo. Int. J. Oncol. 46, 2419-2430. doi:10.3892/ijo.2015.2941

Fujita, K., Kobara, H., Mori, H., Fujihara, S., Chiyo, T., Matsunaga, T., et al. (2015b). Differences in miRNA Expression Profiles Between GIST and Leiomyoma in Human Samples Acquired by Submucosal Tunneling Biopsy. Endosc. Int. Open 03, E665-E671. doi:10.1055/s-0034-1393077

Garcia, D. M., Baek, D., Shin, C., Bell, G. W., Grimson, A., and Bartel, D. P. (2011). Weak Seed-Pairing Stability and High Target-Site Abundance Decrease the Proficiency of Lsy-6 and Other microRNAs. Nat. Struct. Mol. Biol. 18, 1139-1146. doi:10.1038/nsmb.2115

Goodpaster, B. H., Thaete, F. L., and Kelley, D. E. (2000). Thigh Adipose Tissue Distribution Is Associated with Insulin Resistance in Obesity and in Type 2 Diabetes Mellitus. Am. J. Clin. Nutr. 71, 885-892. doi:10.1093/ajcn/71.4.885

Hamam, D., Ali, D., Kassem, M., Aldahmash, A., and Alajez, N. M. (2015). microRNAs as Regulators of Adipogenic Differentiation of Mesenchymal Stem Cells. Stem Cell Dev. 24, 417-425. doi:10.1089/scd.2014.0331

Hocquette, J. F., Gondret, F., Baéza, E., Médale, F., Jurie, C., and Pethick, D. W. (2010). Intramuscular Fat Content in Meat-Producing Animals: Development, Genetic and Nutritional Control, and Identification of Putative Markers. Animal 4, 303-319. doi:10.1017/S1751731109991091

Kato, K., Gong, J., Iwama, H., Kitanaka, A., Tani, J., Miyoshi, H., et al. (2012). The Antidiabetic Drug Metformin Inhibits Gastric Cancer Cell Proliferation In Vitro and In Vivo. Mol. Cancer Ther. 11, 549-560. doi:10.1158/15357163.MCT-11-0594

Kato, K., Iwama, H., Yamashita, T., Kobayashi, K., Fujihara, S., Fujimori, T., et al. (2016). The Anti-Diabetic Drug Metformin Inhibits Pancreatic Cancer Cell Proliferation In Vitro and In Vivo: Study of the microRNAs Associated With the Antitumor Effect of Metformin. Oncol. Rep. 35, 1582-1592. doi:10.3892/ or.2015.4496

\section{FUNDING}

This study was supported by grants from the National Natural Sciences Foundation of China (31672395) and the Applied Basic Research Program Key Project of Sichuan Province (2018JY0036).

Kerr, T. A., Korenblat, K. M., and Davidson, N. O. (2011). MicroRNAs and Liver Disease. Transl. Res. 157, 241-252. doi:10.1038/jhg.2016.5310.1016/ j.trsl.2011.01.008

Kim, M., Park, J.-E., Song, S.-B., and Cha, Y.-S. (2015). Effects of Black Adzuki Bean (Vigna Angularis) Extract on Proliferation and Differentiation of 3T3-L1 Preadipocytes into Mature AdipocytesVigna Angularis) Extract on Proliferation and Differentiation of 3T3-L1 Preadipocytes into Mature Adipocytes. Nutrients 7, 277-292. doi:10.3390/nu7010277

Kinoshita, M., Ono, K., Horie, T., Nagao, K., Nishi, H., Kuwabara, Y., et al. (2010). Regulation of Adipocyte Differentiation by Activation of Serotonin (5-HT) Receptors 5-HT2AR and 5-HT2CR and Involvement of MicroRNA-448Mediated Repression of KLF5. Mol. Endocrinol. 24, 1978-1987. doi:10.1210/ me.2010-0054

Kobayashi, M., Kato, K., Iwama, H., Fujihara, S., Nishiyama, N., Mimura, S., et al. (2013). Antitumor Effect of Metformin in Esophageal Cancer: In Vitro Study. Int. J. Oncol. 42, 517-524. doi:10.3892/ijo.2012.1722

Lee, H., Kim, H. J., Lee, Y. J., Lee, M.-Y., Choi, H., Lee, H., et al. (2012). KrüppelLike Factor KLF8 Plays a Critical Role in Adipocyte Differentiation. Plos One 7, e52474. doi:10.1371/journal.pone.0052474

Lee, H., Kim, J., Park, J. Y., Kang, K. S., Park, J. H., and Hwang, G. S. (2017). Processed Panax Ginseng, Sun Ginseng, Inhibits the Differentiation and Proliferation of 3T3-L1 Preadipocytes and Fat Accumulation in Caenorhabditis elegans. J. Ginseng Res. 41, 257-267. doi:10.1016/ j.jgr.2016.04.004

Li, X.-H., Qu, J.-Q., Yi, H., Zhang, P.-F., Yi, H.-M., Wan, X.-X., et al. (2014). Integrated Analysis of Differential miRNA and mRNA Expression Profiles in Human Radioresistant and Radiosensitive Nasopharyngeal Carcinoma Cells. PLoS One 9, e87767. doi:10.1371/journal.pone.0087767

Li, X., Zhao, Y., Li, X., Wang, Q., Ao, Q., Wang, X., et al. (2019). MicroRNA-150 Modulates Adipogenic Differentiation of Adipose-Derived Stem Cells by Targeting Notch3. Stem Cell Int. 2019, 1-12. doi:10.1155/2019/2743047

Lin, Z., He, H., Wang, M., and Liang, J. (2019). MicroRNA-130a Controls Bone Marrow Mesenchymal Stem Cell Differentiation towards the Osteoblastic and Adipogenic Fate. Cell Prolif 52, e12688. doi:10.1111/cpr.12688

Liu, K., Yu, W., Wei, W., Zhang, X., Tian, Y., Sherif, M., et al. (2019). Melatonin Reduces Intramuscular Fat Deposition by Promoting Lipolysis and Increasing Mitochondrial Function. J. Lipid Res. 60, 767-782. doi:10.1194/ jlr.M087619

Ma, J., Lin, Y., Zhu, J., Huang, K., and Wang, Y. (2021). MiR-26b-5p Regulates the Preadipocyte Differentiation by Targeting FGF21 in Goats. In Vitro Cell.Dev.Biol.-Animal 57, 257-263. doi:10.1007/ s11626-020-00493-y

Maragkakis, M., Alexiou, P., Papadopoulos, G. L., Reczko, M., Dalamagas, T., Giannopoulos, G., et al. (2009). Accurate microRNA Target Prediction Correlates with Protein Repression Levels. BMC Bioinformatics 10, 295. doi:10.1186/1471-2105-10-295

Miyoshi, H., Kato, K., Iwama, H., Maeda, E., Sakamoto, T., Fujita, K., et al. (2014). Effect of the Anti-diabetic Drug Metformin in Hepatocellular Carcinoma In Vitro and In Vivo. Int. J. Oncol. 45, 322-332. doi:10.3892/ijo.2014.2419

Mourelatos, Z. (2008). The Seeds of Silence. Nature 455, 44-45. doi:10.3892/ ijo.2013.223310.1038/455044a

Pei, H., Yao, Y., Yang, Y., Liao, K., and Wu, J.-R. (2011). Krüppel-like Factor KLF9 Regulates PPAR $\gamma$ Transactivation at the Middle Stage of Adipogenesis $\gamma$ Transactivation at the Middle Stage of Adipogenesis. Cell Death Differ 18, 315-327. doi:10.1038/cdd.2010.100

Peng, S.-g., Pang, Y.-1., Zhu, Q., Kang, J.-h., Liu, M.-x., and Wang, Z. (2018). Chlorogenic Acid Functions as a Novel Agonist of PPAR $\gamma 2$ during the Differentiation of Mouse 3T3-L1 Preadipocytes $\gamma 2$ During the Differentiation of Mouse 3T3-L1 Preadipocytes. Biomed. Res. Int. 2018, 8594767. doi:10.1155/ $2018 / 8594767$ 
Redis, R. S., and Calin, G. A. (2017). SnapShot: Non-coding RNAs and Metabolism. Cel Metab. 25, 220. doi:10.1016/j.cmet10.1016/j.cmet.2016.12.012

Rosen, E. D., and MacDougald, O. A. (2006). Adipocyte Differentiation From the Inside Out. Nat. Rev. Mol. Cel Biol. 7, 885-896. doi:10.1038/ nrm2066

Rouleau, S., Glouzon, J.-P. S., Brumwell, A., Bisaillon, M., and Perreault, J.-P. (2017). 3' UTR G-Quadruplexes Regulate miRNA Binding. RNA 23, 1172-1179. doi:10.1261/rna.06096210.1261/rna.060962.117

Schreck, C., Istvánffy, R., Ziegenhain, C., Sippenauer, T., Ruf, F., Henkel, L., et al. (2017). Niche WNT5A Regulates the Actin Cytoskeleton During Regeneration of Hematopoietic Stem Cells. J. Exp. Med. 214, 165-181. doi:10.1084/ jem.20151414

Shen, L., Li, Q., Wang, J., Zhao, Y., Niu, L., Bai, L., et al. (2018). miR-144-3p Promotes Adipogenesis Through Releasing C/EBP $\alpha$ from Klf3 and CtBP2. Front. Genet. 9, 677. doi:10.3389/fgene.2018.00677

Shoelson, S. E., Herrero, L., and Naaz, A. (2007). Obesity, Inflammation, and Insulin Resistance. Gastroenterology 132, 2169-2180. doi:10.1053/ j.gastro10.1053/j.gastro.2007.03.059

Sun, M., Ding, J., Li, D., Yang, G., Cheng, Z., and Zhu, Q. (2017). NUDT21 Regulates $3^{\prime}$-UTR Length and microRNA-Mediated Gene Silencing in Hepatocellular Carcinoma. Cancer Lett. 410, 158-168. doi:10.1016/ j.canlet.2017.09.026

Tada, A., Kober, A. H., Islam, M. A., Igata, M., Takagi, M., Suzuki, M., et al. (2020). Evaluation of Fat Accumulation and Adipokine Production during the LongTerm Adipogenic Differentiation of Porcine Intramuscular Preadipocytes and Study of the Influence of Immunobiotics. Cells 9, 1715. doi:10.3390/ cells 9071715

Tahmasebi, S., Ghorbani, M., Savage, P., Yan, K., Gocevski, G., Xiao, L., et al. (2013). Sumoylation of Krüppel-like Factor 4 Inhibits Pluripotency Induction but Promotes Adipocyte Differentiation. J. Biol. Chem. 288, 12791-12804. doi:10.1074/jbc.M113.465443

Tang, R., Ma, F., Li, W., Ouyang, S., Liu, Z., and Wu, J. (2017). miR-206-3p Inhibits 3T3-L1 Cell Adipogenesis via the C-Met/PI3K/Akt Pathway. Ijms 18, 1510. doi:10.3390/ijms18071510

van der Kwast, R. V. C. T., Quax, P. H. A., and Nossent, A. Y. (2020). An Emerging Role for isomiRs and the microRNA Epitranscriptome in Neovascularization. Cells 9, 61. doi:10.3390/cells9010061
Wong, N., and Wang, X. (2015). miRDB: an Online Resource for microRNA Target Prediction and Functional Annotations. Nucleic Acids Res. 43, D146-D152. doi:10.1093/nar/gku1104

Wood, J. D., Nute, G. R., Richardson, R. I., Whittington, F. M., Southwood, O., Plastow, G., et al. (2004). Effects of Breed, Diet and Muscle on Fat Deposition and Eating Quality in Pigs. Meat Sci. 67, 651-667. doi:10.1016/ j.meatsci.2004.01.007

Xu, Q., Lin, S., Wang, Y., Zhu, J., and Lin, Y. (2018). Fibroblast Growth Factor 10 (FGF10) Promotes the Adipogenesis of Intramuscular Preadipocytes in Goat. Mol. Biol. Rep. 45, 1881-1888. doi:10.1007/s11033-018-4334-1

Xu, Q., Wang, Y., Zhang, Y., Zhu, J., and Lin, Y. (2020). RXRa Cooperates with KLF8 to Promote the Differentiation of Intramuscular Preadipocytes in Goat. Anim. Biotechnol. 27, 1-11. doi:10.1080/10495398.2020.1732397

Yamada, T., Kamiya, M., and Higuchi, M. (2020). Fat Depot-specific Effects of Body Fat Distribution and Adipocyte Size on Intramuscular Fat Accumulation in Wagyu Cattle. Anim. Sci. J. 91, el3449 doi:10.1111/asj.13449

Yan, Z., Guo, Y., Wang, Y., Li, Y., and Wang, J. (2018). MicroRNA Profiles of BMSCs Induced into Osteoblasts with Osteoinductive Medium. Exp. Ther. Med. 15, 2589-2596. doi:10.3892/etm.2018.5723

Conflict of Interest: The authors declare that the research was conducted in the absence of any commercial or financial relationships that could be construed as a potential conflict of interest.

Publisher's Note: All claims expressed in this article are solely those of the authors and do not necessarily represent those of their affiliated organizations, or those of the publisher, the editors and the reviewers. Any product that may be evaluated in this article, or claim that may be made by its manufacturer, is not guaranteed or endorsed by the publisher.

Copyright $\odot 2021 \mathrm{Xu}$, Wang, Li, Du, Li, Zhu and Lin. This is an open-access article distributed under the terms of the Creative Commons Attribution License (CC BY). The use, distribution or reproduction in other forums is permitted, provided the original author(s) and the copyright owner(s) are credited and that the original publication in this journal is cited, in accordance with accepted academic practice. No use, distribution or reproduction is permitted which does not comply with these terms. 